Research Article

\title{
Some Summation Formulas for the Generalized Kampé de Fériet Function
}

\author{
Ashish Verma $\mathbb{D}^{1},{ }^{1}$ Jihad Younis $\mathbb{D}^{2},{ }^{2}$ Vikash Kumar Pandey, ${ }^{3}$ and Hassen Aydi $\mathbb{D}^{4,5,6}$ \\ ${ }^{1}$ Department of Mathematics Prof. Rajendra Singh (Rajju Bhaiya), Institute of Physical Sciences for Study and Research, \\ V. B. S. Purvanchal University, Jaunpur (UP) 222003, India \\ ${ }^{2}$ Department of Mathematics, Aden University, Khormaksar, P. O. Box 6014, Yemen \\ ${ }^{3}$ Department of Mathematics, National PG College, Lucknow (UP) 226001, India \\ ${ }^{4}$ Université de Sousse, Institut Supérieur d'Informatique et des Techniques de Communication, H. Sousse 4000, Tunisia \\ ${ }^{5}$ Department of Mathematics and Applied Mathematics, Sefako Makgatho Health Sciences University, \\ Ga-Rankuwa, South Africa \\ ${ }^{6}$ China Medical University Hospital, China Medical University, Taichung 40402, Taiwan
}

Correspondence should be addressed to Jihad Younis; jihadalsaqqaf@gmail.com and Hassen Aydi; hassen.aydi@isima.rnu.tn

Received 9 June 2021; Accepted 30 August 2021; Published 15 September 2021

Academic Editor: Abdullahi Yusuf

Copyright (c) 2021 Ashish Verma et al. This is an open access article distributed under the Creative Commons Attribution License, which permits unrestricted use, distribution, and reproduction in any medium, provided the original work is properly cited.

The aim of this manuscript is to establish several finite summation formulas (FSFs) for the generalized Kampé de Fériet series (GKDFS). Moreover, the particular result for confluent forms of Lauricella series in $n$ variables and four generalized Lauricella functions are obtained from the finite summation formulas for the GKDFS.

\section{Introduction}

Special functions are essential tools in several equations arising in natural science. The hypergeometric series and its generalizations are appeared in many mathematical problems and their applications. The theory of hypergeometric functions in many variables by the fact that the solutions of partial differential equations appearing in several applied problems of mathematical physics has been presented in terms of such hypergeometric functions [1-4].

Since 2012, Brychkov and Saad [5-8] have obtained many finite summation formulas of Appell's functions $F_{1}$, $F_{2}$, and $F_{3}$. Later, Wang established some infinite summation formulas of double hypergeometric functions [9]. In 2016, Wang and Chen [10] derived FSFs of double hypergeometric functions involving some summation theorems. In 2019, Sahai and Verma [11] gave FSFs for the Srivastava's general triple hypergeometric function [12]. For instance, works of Lauricella functions [13] and Srivastava's triple hypergeometric functions $[14,15]$ have been provided. These works generalized and unified several results in [10] for the three-variable hypergeometric function. In view of the abovementioned works, our motivation is to present here several FSFs for the GKDFS. Also, some particular cases yielding to FSFs for four generalized Lauricella functions and confluent forms of Lauricella series in $n$ variables are given.

The multivariable generalization of Kampé de Fériet function is given as $[2,3]$

$$
\begin{aligned}
& F_{l: v_{1} ; \ldots ; v_{n}}^{p: \mu_{1} ; \ldots ; \mu_{n}}\left[\begin{array}{c}
\left(a_{p}\right):\left(b_{\mu_{1}}^{(1)}\right) ; \ldots ;\left(b_{\mu_{n}}^{(n)}\right) ; \\
\tau_{1}, \ldots, \tau_{n} \\
\left(\alpha_{l}\right):\left(\omega_{v_{1}}^{(1)}\right) ; \ldots ;\left(\omega_{v_{n}}^{(n)}\right) ;
\end{array}\right] \\
& =\sum_{s_{1}, \ldots, s_{n}=0}^{\infty} \wedge\left(s_{1}, e, s_{n}\right) \prod_{i=1}^{n} \frac{\tau_{i}^{s_{i}}}{s_{i} !}
\end{aligned}
$$

where 


$$
\wedge\left(s_{1}, \ldots, s_{n}\right)=\frac{\prod_{j=1}^{p}\left(a_{j}\right)_{s_{1}+\cdots+s_{n}} \prod_{j=1}^{\mu_{1}}\left(b_{j}^{(1)}\right)_{s_{1}} \ldots \prod_{j=1}^{\mu_{n}}\left(b_{j}^{(n)}\right)_{s_{n}}}{\prod_{j=1}^{l}\left(\alpha_{j}\right)_{s_{1}+\cdots+s_{n}} \prod_{j=1}^{v_{1}}\left(\omega_{j}^{(1)}\right)_{s_{1}} \cdots \prod_{j=1}^{v_{n}}\left(\omega_{j}^{(n)}\right)_{s_{n}}},
$$

and, for convergence of (1), $1+l+v_{u}-p-\mu_{u} \geq 0$ for $u=1, \ldots, n$. The equality is satisfied if, in addition, either $\left(p>l\right.$ and $\left.\left|\tau_{1}\right|^{1 /(p-l)}+\cdots+\left|\tau_{n}\right|^{1 /(p-l)}<1\right)$, or $\quad(p \leq l$ and $\left.\max \left(\left|\tau_{1}\right|, \ldots,\left|\tau_{n}\right|\right)<1\right)$.
Next, we give the definition of the derivative operator [3]

$$
D_{\tau} f(\tau)=v_{h \rightarrow 0} \frac{f(\tau+h)-f(\tau)}{h},
$$

provided $f$ is differentiable at $\tau$. Moreover, $D_{\tau}^{n} f(\tau)=$ $D_{\tau}\left(D_{\tau}^{n-1} f(\tau)\right), n=0,1,2, \ldots$

From now, we consider some abbreviated notations:

$$
\begin{aligned}
\left(\theta_{p}+k\right) & =\theta_{1}+k, \ldots, \theta_{p}+k, \\
\left(\theta_{p}^{i}+k\right) & =\theta_{1}+k, \ldots, \theta_{i-1}+k, \theta_{i+1}+k, \ldots, \theta_{p}+k, \quad i=1, \ldots, p, \\
\left(\xi_{\mu_{t}}^{(t)}+k\right) & =\xi_{1}^{(t)}+k, \ldots, \xi_{\mu_{t}}^{(t)}+k, \\
\left(\xi_{\mu_{t}}^{(t), i}+k\right) & =\xi_{1}^{(t)}+k, \ldots, \xi_{i-1}^{(t)}+k, \xi_{i+1}^{(t)}+k, \ldots, \xi_{\mu_{t}}^{(t)}+k, \quad t=1, \ldots, n, 1 \leq i \leq \mu_{t}, \\
{[\theta]_{k} } & =\prod_{j=1}^{p}\left(\theta_{j}\right)_{k}, \\
{\left[\theta^{i}\right]_{k} } & =\prod_{j=1, j \neq i}^{p}\left(\theta_{j}\right)_{k}, \\
{\left[\xi^{(t)}\right]_{k} } & =\prod_{j=1}^{\mu_{t}}\left(\xi_{j}^{(t)}\right)_{k}, \\
{\left[\xi^{(t), i}\right]_{k} } & =\prod_{j=1, j \neq i}^{\mu_{t}}\left(\xi_{j}^{(t)}\right)_{k}, \quad t=1, \ldots, n,
\end{aligned}
$$

where $\left(\theta_{j}\right)_{k}$ corresponds to Pochhammer symbol [16].

\section{FSFs of GKDFS by Derivative Operator}

The FSFs of GKDFS follow by using a derivative operator.

The $r$ th derivative on $\tau_{1}$ of GKDFS is obtained as follows:

$$
\begin{aligned}
& D_{\tau_{1}}^{r}\left\{F_{l: \nu_{1} ; \ldots ; v_{n}}^{p: \nu_{n} ; \mu_{n}}\left[\begin{array}{c}
\left(a_{p}\right):\left(b_{\mu_{1}}^{(1)}\right) ; \ldots ;\left(b_{\mu_{n}}^{(n)}\right) ; \\
\tau_{1}, \ldots, \tau_{n} \\
\left(\alpha_{l}\right):\left(\omega_{v_{1}}^{(1)}\right) ; \ldots ;\left(\omega_{v_{n}}^{(n)}\right) ;
\end{array}\right]\right\} \\
& =\frac{[a]_{r}\left[b^{(1)}\right]_{r}}{[\alpha]_{r}\left[\omega^{(1)}\right]_{r}} F_{l: v_{1} ; \ldots ; v_{n}}^{p: \mu_{1} ; \ldots ; \mu_{n}}\left[\begin{array}{l}
\left(a_{p}+r\right):\left(b_{\mu_{1}}^{(1)}+r\right) ;\left(b_{\mu_{2}}^{(2)}\right) ; \ldots ;\left(b_{\mu_{n}}^{(n)}\right) ; \\
\tau_{1}, \ldots, \tau_{n} \\
\left(\alpha_{l}+r\right):\left(\omega_{v_{1}}^{(1)}+r\right) ;\left(\omega_{v_{2}}^{(2)}\right) ; \ldots ;\left(\omega_{v_{n}}^{(n)}\right) ;
\end{array}\right] .
\end{aligned}
$$

Due to the Leibnitz formula

$$
D_{\tau_{1}}^{r}\left(f\left(\tau_{1}\right) g\left(\tau_{1}\right)\right)=\sum_{k=0}^{r}\left(\begin{array}{l}
r \\
k
\end{array}\right) D_{\tau_{1}}^{r-k} f\left(\tau_{1}\right) D_{\tau_{1}}^{k} g\left(\tau_{1}\right),
$$

and (5), the following FSFs of GKDFS follow.

Theorem 1. We have the following FSFs of GKDFS: 


$$
\begin{aligned}
& \sum_{k=0}^{r}\left(\begin{array}{c}
r \\
k
\end{array}\right) \frac{\left[a^{i}\right]_{k}\left[b^{(1)}\right]_{k}}{[\alpha]_{k}\left[\omega^{(1)}\right]_{k}} \tau_{1}^{k} F_{l: v_{1} ; \ldots ; \nu_{n}}^{p: \mu_{1} ; \ldots ; \mu_{n}}\left[\begin{array}{c}
\left(a_{p}+k\right):\left(b_{\mu_{1}}^{(1)}+k\right) ;\left(b_{\mu_{2}}^{(2)}\right) ; \ldots ;\left(b_{\mu_{n}}^{(n)}\right) ; \\
\left(\alpha_{l}+k\right):\left(\omega_{v_{1}}^{(1)}+k\right) ;\left(\omega_{v_{2}}^{(2)}\right) ; \ldots ;\left(\omega_{v_{n}}^{(n)}\right) ;
\end{array}\right] \\
& \quad=F_{l: v_{1}+1 ; v_{2} ; \ldots ; v_{n}}^{p: \mu_{1}+1 ; \mu_{2} ; \ldots ; \mu_{n}}\left[\begin{array}{c}
\left(a_{p}\right): a_{i}+r,\left(b_{\mu_{1}}^{(1)}\right) ;\left(b_{\mu_{2}}^{(2)}\right) ; \ldots ;\left(b_{\mu_{n}}^{(n)}\right) ; \\
\left(\alpha_{l}\right): a_{i},\left(\omega_{v_{1}}^{(1)}\right) ;\left(\omega_{v_{2}}^{(2)}\right) ; \ldots ;\left(\omega_{v_{n}}^{(n)}\right) ;
\end{array}\right]
\end{aligned}
$$

with $i=1, \ldots, p$;

$$
\begin{aligned}
& \sum_{k=0}^{r}\left(\begin{array}{c}
r \\
k
\end{array}\right) \frac{[a]_{k}\left[b^{(1), i}\right]_{r}}{[\alpha]_{k}\left[\omega^{(1)}\right]_{r}^{k}} \tau_{1}^{k} F_{l: v_{1} ; \ldots ; v_{n}}^{p: \ldots ; \mu_{n}}\left[\begin{array}{c}
\left(a_{p}+k\right):\left(b_{\mu_{1}}^{(1)}+k\right) ;\left(b_{\mu_{2}}^{(2)}\right) ; \ldots ;\left(b_{\mu_{n}}^{(n)}\right) ; \\
\left.\tau_{1}, \ldots, \tau_{n}\right] \\
\left(\alpha_{l}+k\right):\left(\omega_{v_{1}}^{(1)}+k\right) ;\left(\omega_{v_{2}}^{(2)}\right) ; \ldots ;\left(\omega_{v_{n}}^{(n)}\right) ;
\end{array}\right] \\
& \quad=F_{l: v_{1}+1 ; v_{2} ; \ldots ; v_{n}}^{p: \mu_{1}+1 ; \mu_{2} ; \ldots ; \mu_{n}}\left[\begin{array}{c}
\left.\left(a_{p}\right): a_{i}^{(1)}+r,\left(b_{\mu_{1}}^{(1), i}\right) ;\left(b_{\mu_{2}}^{(2)}\right) ; \ldots ;\left(b_{\mu_{n}}^{(n)}\right) ; \tau_{1}, \ldots, \tau_{n}\right], \\
\left(\alpha_{l}\right):\left(\omega_{v_{1}}^{(1)}\right) ;\left(\omega_{v_{2}}^{(2)}\right) ; \ldots ;\left(\omega_{v_{n}}^{(n)}\right) ;
\end{array}\right.
\end{aligned}
$$

with $i=1, \ldots, \mu_{1}$.

Proof. We prove the identity (7). Using the definition of GKDFS and the Leibnitz formula for differentiation of a product of two functions, one writes

$$
\begin{aligned}
& D_{\tau_{1}}^{r}\left\{\tau_{1}^{a_{i}+r-1} F_{l: v_{1} ; \ldots ; v_{n}}^{p: \mu_{1} ; \ldots ; \mu_{n}}\left[\begin{array}{l}
\left(a_{p}\right):\left(b_{\mu_{1}}^{(1)}\right) ; \ldots ;\left(b_{\mu_{n}}^{(n)}\right) ; \\
\tau_{1}, \ldots, \tau_{n} \\
\left(\alpha_{l}\right):\left(\omega_{v_{1}}^{(1)}\right) ; \ldots ;\left(\omega_{v_{n}}^{(n)}\right) ;
\end{array}\right]\right\} \\
& =\sum_{k=0}^{r}\left(\begin{array}{c}
r \\
k
\end{array}\right) D_{\tau_{1}}^{r-k}\left\{\tau_{1}^{a_{i}+r-1}\right\} D_{\tau_{1}}^{k}\left\{F_{l: v_{1} ; \ldots ; v_{n}}^{p: \mu_{1} ; \ldots ; \mu_{n}}\left[\begin{array}{c}
\left(a_{p}\right):\left(b_{\mu_{1}}^{(1)}\right) ; \ldots ;\left(b_{\mu_{n}}^{(n)}\right) ; \\
\tau_{1}, \ldots, \tau_{n} \\
\left(\alpha_{l}\right):\left(\omega_{v_{1}}^{(1)}\right) ; \ldots ;\left(\omega_{v_{n}}^{(n)}\right) ;
\end{array}\right]\right\} \\
& =\left(a_{i}\right)_{r} \tau_{1}^{a_{i}-1} \sum_{k=0}^{r}\left(\begin{array}{l}
r \\
k
\end{array}\right) \frac{\left[a^{i}\right]_{k}\left[b^{(1)}\right]_{k}}{[\alpha]_{k}\left[\omega^{(1)}\right]_{k}^{k}} \\
& \times F_{l: v_{1} ; \ldots ; v_{n}}^{p: \mu_{1} ; \ldots ; \mu_{n}}\left[\begin{array}{c}
\left(a_{p}+k\right):\left(b_{\mu_{1}}^{(1)}+k\right) ;\left(b_{\mu_{2}}^{(2)}\right) ; \ldots ;\left(b_{\mu_{n}}^{(n)}\right) ; \\
\tau_{1}, \ldots, \tau_{n} \\
\left(\alpha_{l}+k\right):\left(\omega_{v_{1}}^{(1)}+k\right) ;\left(\omega_{v_{2}}^{(2)}\right) ; \ldots ;\left(\omega_{v_{n}}^{(n)}\right) ;
\end{array}\right] .
\end{aligned}
$$

Here, we used (5) and a simplification in the second equality. Again, combine $\tau_{1}^{a_{i}+r-1}$ with the variable $\tau_{1}$ in the
GKDFS and put the derivative operator $r$-times on $\tau_{1}$ to get the following: 


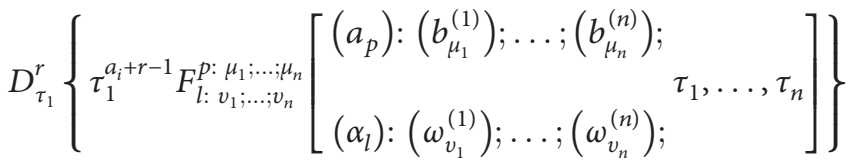

$$
\begin{aligned}
& =\sum_{\varsigma_{1}, \ldots, \varsigma_{n}=0}^{\infty} \wedge\left(\varsigma_{1}, \ldots, \varsigma_{n}\right)\left(a_{i}+\varsigma_{1}\right)_{r} \tau_{1}^{a_{i}-1} \prod_{i=1}^{n} \frac{\tau_{i}^{\varsigma_{i}}}{\varsigma_{i} !} \\
& =\left(a_{i}\right)_{r} \tau_{1}^{a_{i}-1} F_{l: v_{1}+1 ; v_{2} ; \ldots ; v_{n}}^{p: \mu_{1}+1 ; \mu_{n} ; \mu_{n}}\left[\begin{array}{c}
\left(a_{p}\right): a_{i}+r,\left(b_{\mu_{1}}^{(1)}\right) ;\left(b_{\mu_{2}}^{(2)}\right) ; \ldots ;\left(b_{\mu_{n}}^{(n)}\right) ; \\
\left(\alpha_{l}\right): a_{i},\left(\omega_{v_{1}}^{(1)}\right) ;\left(\omega_{v_{2}}^{(2)}\right) ; \ldots ;\left(\omega_{v_{n}}^{(n)}\right) ;
\end{array}\right] .
\end{aligned}
$$

Formula (7) follows clearly. The proof of (8) is done similarly.

Theorem 2. We have the following FSF of GKDFS:

$$
\begin{aligned}
& \sum_{k=0}^{r}\left(\begin{array}{c}
r \\
k
\end{array}\right) \frac{[a]_{k}\left[b^{(1)}\right]_{k}}{\left(\omega_{i}^{(1)}-r\right)_{k}[\alpha]_{k}\left[\omega^{(1)}\right]_{k}} \tau_{1}^{k} F_{l: v_{1} ; \ldots ; v_{n}}^{p: \mu_{1} ; \ldots ; \mu_{n}}\left[\begin{array}{c}
\left(a_{p}+k\right):\left(b_{\mu_{1}}^{(1)}+k\right) ;\left(b_{\mu_{2}}^{(2)}\right) ; \ldots ;\left(b_{\mu_{n}}^{(n)}\right) ; \\
\left(\alpha_{l}+k\right):\left(\omega_{v_{1}}^{(1)}+k\right) ;\left(\omega_{v_{2}}^{(2)}\right) ; \ldots ;\left(\omega_{v_{n}}^{(n)}\right) ;
\end{array}\right] \\
& \quad=F_{l: v_{1} ; \ldots ; v_{n}}^{p: \mu_{1} ; \ldots ; \mu_{n}}\left[\begin{array}{c}
\left.\left(a_{p}\right):\left(b_{\mu_{1}}^{(1)}\right) ;\left(b_{\mu_{2}}^{(2)}\right) ; \ldots ;\left(b_{\mu_{n}}^{(n)}\right) ; \quad \tau_{n}, \ldots, \tau_{n}\right], \\
\left(\alpha_{l}\right): \omega_{i}^{(1)}-r,\left(\omega_{v_{1}}^{(1), i}\right) ;\left(\omega_{v_{2}}^{(2)}\right) ; \ldots ;\left(\omega_{v_{n}}^{(n)}\right) ;
\end{array}\right]
\end{aligned}
$$

where $i=1, \ldots, v_{1}$.

Theorem 3. We have the following FSFs of GKDFS:

Proof. As in the proof of Theorem 1, the application of the derivative operator $r$-times on $\tau_{1}^{\omega_{i}^{(1)}-1} F_{l: v_{1} ; \ldots ; v_{n}}^{p: \mu_{1} ; \ldots ; \mu_{n}}\left(\tau_{1}, \ldots, \tau_{n}\right)$ yields (11). We omit the details.

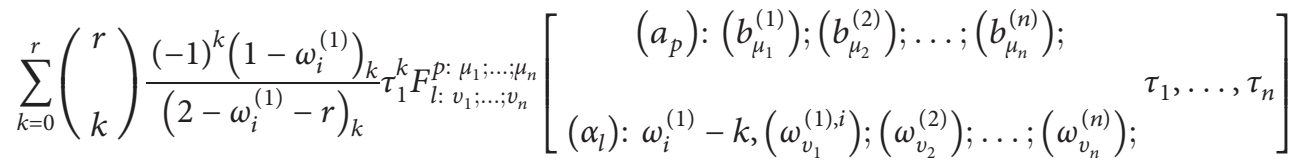

$$
\begin{aligned}
& =\frac{(-1)^{r}[a]_{r}\left[b^{(1)}\right]_{r}}{\left(\omega_{i}^{(1)}-1\right)_{r}[\alpha]_{r}\left[\omega^{(1)}\right]_{r}} F_{l: v_{1} ; \ldots ; v_{n}}^{p: \mu_{1} ; ; \mu_{n}}\left[\begin{array}{c}
\left(a_{p}+r\right):\left(b_{\mu_{1}}^{(1)}+r\right) ;\left(b_{\mu_{2}}^{(2)}\right) ; \ldots ;\left(b_{\mu_{n}}^{(n)}\right) ; \\
\tau_{1}, \ldots, \tau_{n} \\
\left(\alpha_{l}+r\right):\left(\omega_{v_{1}}^{(1)}+r\right) ;\left(\omega_{v_{2}}^{(2)}\right) ; \ldots ;\left(\omega_{v_{n}}^{(n)}\right) ;
\end{array}\right] \\
& \sum_{k=0}^{r}\left(\begin{array}{l}
r \\
k
\end{array}\right) \frac{(-1)^{k}\left(\omega_{i}^{(1)}+r-1\right)_{k}}{\left(\omega_{i}^{(1)}\right)_{k}} \tau_{1}^{k} F_{l: v_{1} ; \ldots ; v_{n}}^{p: \mu_{1} ; \ldots ; \mu_{n}}\left[\begin{array}{c}
\left(a_{p}\right):\left(b_{\mu_{1}}^{(1)}\right) ;\left(b_{\mu_{2}}^{(2)}\right) ; \ldots ;\left(b_{\mu_{n}}^{(n)}\right) ; \\
\left(\alpha_{l}\right): \omega_{i}^{(1)}+k,\left(\omega_{v_{1}}^{(1), i}\right) ;\left(\omega_{v_{2}}^{(2)}\right) ; \ldots ;\left(\omega_{v_{n}}^{(n)}\right) ;
\end{array} \tau_{1}, \ldots, \tau_{n}\right] \\
& =\frac{[a]_{r}\left[b^{(1)}\right]_{r}}{\left(\omega_{i}^{(1)}+r\right)_{r}[\alpha]_{r}\left[\omega^{(1)}\right]_{r}} \tau_{1}^{r} F_{l: v_{1} ; \ldots ; v_{n}}^{p: \mu_{1} ; \ldots ; \mu_{n}}\left[\begin{array}{c}
\left(a_{p}+r\right):\left(b_{\mu_{1}}^{(1)}+r\right) ;\left(b_{\mu_{2}}^{(2)}\right) ; \ldots ;\left(b_{\mu_{n}}^{(n)}\right) ; \\
\left(\alpha_{l}+r\right): \omega_{i}^{(1)}+2 r,\left(\omega_{v_{1}}^{(1), i}+r\right) ;\left(\omega_{v_{2}}^{(2)}\right) ; \ldots ;\left(\omega_{v_{n}}^{(n)}\right) ;
\end{array} \tau_{1}, \ldots, \tau_{n}\right],
\end{aligned}
$$

where $i=1, \ldots, v_{1}$. 
Proof. We first establish (12). Due to GKDFS and the Leibnitz formula for differentiation of a product of two functions, one gets

$$
\begin{aligned}
& D_{\tau_{1}}^{r}\left\{\tau_{1}^{1-\omega_{i}^{(1)}} \times \tau_{1}^{1-\omega_{i}^{(1)}} F_{l: v_{1} ; \ldots ; v_{n}}^{p: \mu_{1} ; \ldots ; \mu_{n}}\left[\begin{array}{l}
\left(a_{p}\right):\left(b_{\mu_{1}}^{(1)}\right) ; \ldots ;\left(b_{\mu_{n}}^{(n)}\right) ; \\
\left(\alpha_{l}\right):\left(\omega_{v_{1}}^{(1)}\right) ; \ldots ;\left(\omega_{v_{n}}^{(n)}\right) ;
\end{array}\right]\right\} \\
& =\sum_{k=0}^{r}\left(\begin{array}{c}
r \\
k
\end{array}\right) D_{\tau_{1}}^{r-k}\left\{\tau_{1}^{1-\omega_{i}^{(1)}}\right\} D_{\tau_{1}}^{k}\left\{\tau_{1}^{\omega_{i}^{(1)}-1} F_{l: v_{1} ; \ldots ; v_{n}}^{p: \mu_{1} ; \mu_{n}}\left[\begin{array}{c}
\left(a_{p}\right):\left(b_{\mu_{1}}^{(1)}\right) ; \ldots ;\left(b_{\mu_{n}}^{(n)}\right) ; \\
\left.\tau_{1}, \ldots, \tau_{n}\right):\left(\omega_{v_{1}}^{(1)}\right) ; \ldots ;\left(\omega_{v_{n}}^{(n)}\right) ;
\end{array}\right]\right\} \\
& =\sum_{k=0}^{r}(-1)^{r+k}\left(\begin{array}{c}
r \\
k
\end{array}\right) \frac{\left(\omega_{i}^{(1)}-1\right)_{r}\left(1-\omega_{i}^{(1)}\right)_{k}}{\left(2-\omega_{i}^{(1)}-r\right)_{k} \tau_{1}^{r}} \times F_{l: v_{1} ; \ldots ; v_{n}}^{p: \mu_{1} ; \ldots ; \mu_{n}}\left[\begin{array}{c}
\left(a_{p}\right):\left(b_{\mu_{1}}^{(1)}+k\right) ;\left(b_{\mu_{2}}^{(2)}\right) ; \ldots ;\left(b_{\mu_{n}}^{(n)}\right) ; \\
\left(\alpha_{l}\right): \omega_{i}^{(1)}-k,\left(\omega_{v_{1}}^{(1), i}\right) ;\left(\omega_{v_{2}}^{(2)}\right) ; \ldots ;\left(\omega_{v_{n}}^{(n)}\right) ; \\
\tau_{1}, \ldots, \tau_{n}
\end{array}\right] .
\end{aligned}
$$

The use of the derivative operator $r$-times on GKDFS and the combination with the above lead to (12). The application of $D_{\tau_{1}}^{r}$ on

$$
\tau_{1}^{1-\omega_{i}^{(1)}-r} \times \tau_{1}^{\omega_{i}^{(1)}+r-1} F_{l: v_{1} ; \ldots ; v_{n}}^{p: \mu_{1} ; \ldots ; \mu_{n}}\left[\begin{array}{c}
\left(a_{p}\right):\left(b_{\mu_{1}}^{(1)}+r\right) ;\left(b_{\mu_{2}}^{(2)}\right) ; \ldots ;\left(b_{\mu_{n}}^{(n)}\right) ; \\
\left(\alpha_{l}\right): \omega_{i}^{(1)}+r,\left(\omega_{v_{1}}^{(1), i}\right) ;\left(\omega_{v_{2}}^{(2)}\right) ; \ldots ;\left(\omega_{v_{n}}^{(n)}\right) ;
\end{array} \tau_{1}, \ldots, \tau_{n}\right] .
$$

Formula (13) follows as in the proof of (12).

Theorem 4. We have the following FSFs of GKDFS:

$$
\begin{gathered}
\sum_{k=0}^{r} \frac{(-r)_{k}}{\left(a_{i}-r+1\right)_{k}} F_{l+1: v_{1}: \ldots: v_{n}}^{p+1: \mu_{1}: \ldots: \mu_{n}}\left[\begin{array}{c}
\left.1+k,\left(a_{p}\right):\left(b_{\mu_{i}}^{(1)}\right) ; \ldots ;\left(b_{\mu_{n}}^{(1)}\right) ; \frac{1}{\tau_{1}}, \ldots \frac{1}{\tau_{1}}\right] \\
1,\left(\alpha_{l}\right):\left(\omega_{v_{i}}^{(1)}\right) ; \ldots ;\left(\omega_{v_{n}}^{(1)}\right) ;
\end{array}\right. \\
\quad=\frac{a_{i}-r}{a_{i}} F_{l+1: v_{1}: \ldots: v_{n}}^{p+1: \mu_{1}: \ldots: \mu_{n}}\left[\begin{array}{c}
1-a_{i}+r,\left(a_{p}\right):\left(b_{\mu_{i}}^{(1)}\right) ; \ldots ;\left(b_{\mu_{n}}^{(1)}\right) ; \frac{1}{\tau_{1}}, \ldots \frac{1}{\tau_{1}} \\
1-a_{i},\left(\alpha_{l}\right):\left(\omega_{v_{i}}^{(1)}\right) ; \ldots ;\left(\omega_{v_{n}}^{(1)}\right) ;
\end{array}\right.
\end{gathered}
$$

with $i=1, \ldots, p$;

$$
\begin{aligned}
& \sum_{k=0}^{r} \frac{(-r)_{k}}{\left(b_{i}^{(1)}-r+1\right)_{k}} F_{l: v_{1}+1 ; v_{2} ; \ldots ; v_{n}}^{p: \mu_{1}+1 ; \mu_{2} ; \ldots ; \mu_{n}}\left[\begin{array}{c}
\left.\left(a_{p}\right): 1+k,\left(b_{\mu_{1}}^{(1)}\right) ; \ldots ;\left(b_{\mu_{n}}^{(n)}\right) ; \frac{1}{\tau_{1}}, \tau_{2}, \ldots, \tau_{n}\right] \\
\left(\alpha_{l}\right): 1,\left(\omega_{v_{1}}^{(1)}\right) ; \ldots ;\left(\omega_{v_{n}}^{(n)}\right) ;
\end{array}\right. \\
& \quad=\frac{b_{i}^{(1)}-r}{b_{i}^{(1)}} F_{l: v_{1}+1 ; v_{2} ; \ldots ; v_{n}}^{p: \mu_{1}+1 ; \mu_{2} ; \ldots ; \mu_{n}}\left[\begin{array}{c}
\left(a_{p}\right): 1-b_{i}^{(1)}+r,\left(b_{\mu_{1}}^{(1)}\right) ; \ldots ;\left(b_{\mu_{n}}^{(n)}\right) ; \frac{1}{\tau_{1}}, \tau_{2}, \ldots, \tau_{n} \\
\left(\alpha_{l}\right): 1-b_{i}^{(1)},\left(\omega_{v_{1}}^{(1)}\right) ; \ldots ;\left(\omega_{v_{n}}^{(n)}\right) ;
\end{array}\right],
\end{aligned}
$$

with $i=1, \ldots, \mu_{1}$. 
Proof. We first establish (16). Note that formula (17) could be done similarly. The calculation of $r$ th derivatives on $\tau_{1}$ of $\tau_{1}^{a_{i}-1} F_{l: v_{1} ; \ldots ; v_{n}}^{p: \mu_{1} ; \ldots ; \mu_{n}}\left(1 / \tau_{1}, \ldots, 1 / \tau_{n}\right)$ gives

$$
\begin{aligned}
& D_{\tau_{1}}^{r}\left\{\tau_{1}^{a_{i}-1} F_{l: v_{1} ; \ldots ; v_{n}}^{p: \mu_{1} ; \ldots ; v_{n}}\left[\begin{array}{l}
\left(a_{p}\right):\left(b_{\mu_{1}}^{(1)}\right) ; \ldots ;\left(b_{\mu_{n}}^{(n)}\right) ; \frac{1}{\tau_{1}}, \ldots, \frac{1}{\tau_{1}} \\
\left(\alpha_{l}\right):\left(\omega_{v_{1}}^{(1)}\right) ; \ldots ;\left(\omega_{v_{n}}^{(n)}\right) ;
\end{array}\right\}\right. \\
& =(-1)^{r} \frac{\left(1-a_{i}\right)_{r}}{\tau_{1}^{r+1-a_{i}}} F_{l+1: v_{1} ; \ldots ; v_{n}}^{p+1: \mu_{1} ; \ldots ; \mu_{n}}\left[\begin{array}{c}
1-a_{i}+r,\left(a_{p}\right):\left(b_{\mu_{1}}^{(1)}\right) ; \ldots ;\left(b_{\mu_{n}}^{(n)}\right) ; \\
1-a_{i},\left(\alpha_{l}\right):\left(\omega_{v_{1}}^{(1)}\right) ; \ldots ;\left(\omega_{v_{n}}^{(n)}\right) ;
\end{array} \frac{1}{\tau_{1}}, \ldots, \frac{1}{\tau_{1}}\right] .
\end{aligned}
$$

Due to the Leibnitz formula, one has

$$
\begin{aligned}
& D_{\tau_{1}}^{r}\left\{\tau_{1}^{a_{i}-1} F_{l: v_{1} ; \ldots ; v_{n}}^{p: \mu_{1} ; \ldots ; \mu_{n}}\left[\begin{array}{l}
\left(a_{p}\right):\left(b_{\mu_{1}}^{(1)}\right) ; \ldots ;\left(b_{\mu_{n}}^{(n)}\right) ; \frac{1}{\tau_{1}}, \ldots, \frac{1}{\tau_{1}} \\
\left(\alpha_{l}\right):\left(\omega_{v_{1}}^{(1)}\right) ; \ldots ;\left(\omega_{v_{n}}^{(n)}\right) ;
\end{array}\right\}\right. \\
& =\sum_{k=0}^{r}\left(\begin{array}{c}
r \\
k
\end{array}\right) D_{\tau_{1}}^{r-k}\left\{\tau_{1}^{a_{i}}\right\} D_{\tau_{1}}^{k}\left\{\tau_{1}^{-1} F_{l: v_{1} ; \ldots ; v_{n}}^{p: \mu_{1} ; \ldots ; \mu_{n}}\left[\begin{array}{c}
\left(a_{p}\right):\left(b_{\mu_{1}}^{(1)}\right) ; \ldots ;\left(b_{\mu_{n}}^{(n)}\right) ; \\
\frac{1}{\tau_{1}}, \ldots, \frac{1}{\tau_{1}}
\end{array}\right]\right\}
\end{aligned}
$$

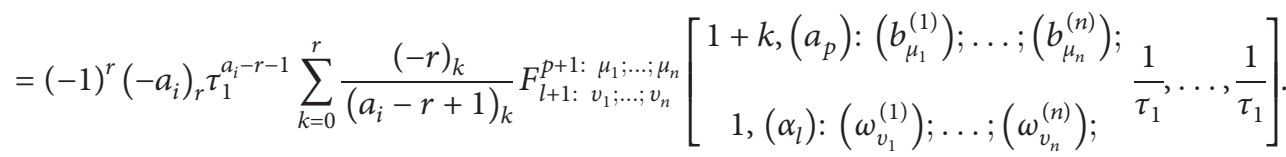

With the combination of the above, one has (16).

Theorem 5. The following FSFs of GKDFS are satisfied:

$$
\begin{aligned}
& \sum_{k=0}^{r} \frac{(-r)_{k}}{\left(2-a_{i}-r\right)_{k}} F_{l+1: v_{1} ; \ldots ; v_{n}}^{p+1: \mu_{n} ; \ldots ; \mu_{n}}\left[\begin{array}{c}
1+k,\left(a_{p}^{i}\right):\left(b_{\mu_{1}}^{(1)}\right) ; \ldots ;\left(b_{\mu_{n}}^{(n)}\right) ; \\
1,\left(\alpha_{l}\right):\left(\omega_{v_{1}}^{(1)}\right) ; \ldots ;\left(\omega_{v_{n}}^{(n)}\right) ;
\end{array} \frac{1}{\tau_{1}}, \ldots, \frac{1}{\tau_{1}}\right] \\
& =\frac{a_{i}+r-1}{a_{i}-1} F_{l: v_{1} ; \ldots ; v_{n}}^{p: \mu_{n} ; \mu_{n}}\left[\begin{array}{c}
a_{i}+r,\left(a_{p}^{i}\right):\left(b_{\mu_{1}}^{(1)}\right) ; \ldots ;\left(b_{\mu_{n}}^{(n)}\right) ; \\
\left(\alpha_{l}\right):\left(\omega_{v_{1}}^{(1)}\right) ; \ldots ;\left(\omega_{v_{n}}^{(n)}\right) ;
\end{array} \frac{1}{\tau_{1}}, \ldots, \frac{1}{\tau_{1}}\right],
\end{aligned}
$$

with $i=1, \ldots, p$;

$$
\begin{gathered}
\sum_{k=0}^{r} \frac{(-r)_{k}}{\left(2-b_{i}^{(1)}-r\right)_{k}} F_{l+1: v_{1} ; \ldots ; v_{n}}^{p+1: \mu_{1} ; \ldots ;}\left[\begin{array}{c}
\left(a_{p}\right): 1+k,\left(b_{\mu_{1}}^{(1)}+k\right) ; \ldots ;\left(b_{\mu_{n}}^{(n)}\right) ; \frac{1}{\tau_{1}}, \tau_{2}, \ldots, \tau_{n} \\
\left(\alpha_{l}\right): 1,\left(\omega_{v_{1}}^{(1)}+k\right) ; \ldots ;\left(\omega_{v_{n}}^{(n)}\right) ;
\end{array}\right] \\
=\frac{b_{i}^{(1)}+r-1}{b_{i}^{(1)}-1} F_{l: v_{1} ; \ldots ; v_{n}}^{p: \mu_{1} ; \ldots ; \mu_{n}}\left[\begin{array}{c}
\left(a_{p}\right): b_{i}^{(1)}+r,\left(b_{\mu_{1}}^{(1)}\right) ; \ldots ;\left(b_{\mu_{n}}^{(n)}\right) ; \frac{1}{\tau_{1}}, \tau_{2}, \ldots, \tau_{n} \\
\left(\alpha_{l}\right):\left(\omega_{v_{1}}^{(1)}\right) ; \ldots ;\left(\omega_{v_{n}}^{(n)}\right) ;
\end{array}\right.
\end{gathered}
$$


with $i=1, \ldots, \mu_{1}$.

Proof. We multiply the L. H. S of $F_{l: v_{1} ; \ldots ; v_{n}}^{p: \mu_{1} ; \ldots ; \mu_{n}}\left(1 / \tau_{1}, \ldots, 1 / \tau_{n}\right)$ series by $\tau_{1}^{1-a_{i}} \times \tau_{1}^{-1}$ and we use the derivative operator as in
(16) to prove (20). The proof of formula (21) is done similarly.

Theorem 6. The following FSFs of GKDFS are verified:

$$
\begin{aligned}
& \sum_{k=0}^{r} \frac{(-r)_{k}}{(2-2 r)_{k}} F_{l+1: v_{1} ; \ldots ; v_{n}}^{p+1: \mu_{1} ; \ldots \mu_{n}}\left[\begin{array}{c}
1+k,\left(a_{p}\right):\left(b_{\mu_{1}}^{(1)}\right) ; \ldots ;\left(b_{\mu_{n}}^{(n)}\right) ; \\
1,\left(\alpha_{l}\right):\left(\omega_{v_{1}}^{(1)}\right) ; \ldots ;\left(\omega_{v_{n}}^{(n)}\right) ;
\end{array} \frac{1}{\tau_{1}}, \ldots, \frac{1}{\tau_{1}}\right] \\
& =\frac{2 r-1}{r-1} F_{l+1: v_{1} ; \ldots ; v_{n}}^{p+1: \mu_{1} ; \ldots ;}\left[\begin{array}{c}
2 r,\left(a_{p}\right): b_{i}^{(1)}+r,\left(b_{\mu_{1}}^{(1)}\right) ; \ldots ;\left(b_{\mu_{n}}^{(n)}\right) ; \\
r,\left(\alpha_{l}\right):\left(\omega_{v_{1}}^{(1)}\right) ; \ldots ;\left(\omega_{v_{n}}^{(n)}\right) ;
\end{array}\right], \ldots, \frac{1}{\tau_{1}}, \ldots
\end{aligned}
$$

with $i=1, \ldots, p$;

$$
\begin{gathered}
\sum_{k=0}^{r} \frac{(-r)_{k}}{(2-2 r)_{k}} s F_{l: v_{1}+1 ; v_{2} ; \ldots ; v_{n}}^{p: \mu_{1}+1 ; \mu_{2} ; \ldots ; \mu_{n}}\left[\begin{array}{c}
\left(a_{p}\right): 1+k,\left(b_{\mu_{1}}^{(1)}\right) ; \ldots ;\left(b_{\mu_{n}}^{(n)}\right) ; \frac{1}{\tau_{1}}, \tau_{2}, \ldots, \tau_{n} \\
\left(\alpha_{l}\right): 1,\left(\omega_{v_{1}}^{(1)}\right) ; \ldots ;\left(\omega_{v_{n}}^{(n)}\right) ;
\end{array}\right] \\
\left.\quad=\frac{2 r-1}{r-1} F_{l: v_{1}+1 ; \nu_{2} ; \ldots ; v_{n}}^{p: \mu_{1}+1 ; \mu_{2} ; \ldots ; \mu_{n}}\left[\begin{array}{c}
\left(a_{p}\right): 2 r,\left(b_{\mu_{1}}^{(1)}\right) ; \ldots ;\left(b_{\mu_{n}}^{(n)}\right) ; \\
\left(\alpha_{l}\right): r,\left(\omega_{v_{1}}^{(1)}\right) ; \ldots ;\left(\omega_{v_{n}}^{(n)}\right) ;
\end{array}\right], \frac{1}{\tau_{1}}, \tau_{2}, \ldots, \tau_{n}\right],
\end{gathered}
$$

with $i=1, \ldots, \mu_{1}$.

Proof. We first present an idea to establish (22). We multiply the L. H. S of $F_{l: v_{1} ; \ldots \ldots ; v_{n}}^{p: \mu_{1} ; \ldots ; \mu_{n}}\left(1 / \tau_{1}, \ldots, 1 / \tau_{n}\right)$-series by $\tau_{1}^{1-r} \times \tau_{1}^{-1}$ and we apply the derivative operator on as in the proof of
(16) to obtain (22). Formula (23) may be done in a similar strategy.

Theorem 7. The following FSFs of GKDFS are verified:

$$
\begin{aligned}
& \sum_{k=0}^{r} \frac{(-r)_{k}}{(1-2 r)_{k}} F_{l+1: v_{1}, \ldots ; v_{n}}^{p+1: \mu_{1} ; \ldots ;}\left[\begin{array}{c}
1+k,\left(a_{p}\right):\left(b_{\mu_{1}}^{(1)}\right) ; \ldots ;\left(b_{\mu_{n}}^{(n)}\right) ; \\
1,\left(\alpha_{l}\right):\left(\omega_{v_{1}}^{(1)}\right) ; \ldots ;\left(\omega_{v_{n}}^{(n)}\right) ;
\end{array} \frac{1}{\tau_{1}}, \ldots, \frac{1}{\tau_{1}}\right] \\
& =2 F_{l+1: v_{1} ; \ldots ; v_{n}}^{p+1: \mu_{1} \ldots ; \mu_{n}}\left[\begin{array}{c}
1+2 r,\left(a_{p}\right): b_{i}^{(1)}+r,\left(b_{\mu_{1}}^{(1)}\right) ; \ldots ;\left(b_{\mu_{n}}^{(n)}\right) ; \\
1+r,\left(\alpha_{l}\right):\left(\omega_{v_{1}}^{(1)}\right) ; \ldots ;\left(\omega_{v_{n}}^{(n)}\right) ;
\end{array} \quad \frac{1}{\tau_{1}}, \ldots, \frac{1}{\tau_{1}}\right] \text {, } \\
& \sum_{k=0}^{r} \frac{(-r)_{k}}{(1-2 r)_{k}} F_{l: v_{1}+1 ; v_{2} ; \ldots ; v_{n}}^{p: \mu_{1}+1 ; \mu_{2} ; \ldots ; \mu_{n}}\left[\begin{array}{c}
\left(a_{p}\right): 1+k,\left(b_{\mu_{1}}^{(1)}\right) ; \ldots ;\left(b_{\mu_{n}}^{(n)}\right) ; \frac{1}{\tau_{1}}, \tau_{2}, \ldots, \tau_{n} \\
\left(\alpha_{l}\right): 1,\left(\omega_{v_{1}}^{(1)}\right) ; \ldots ;\left(\omega_{v_{n}}^{(n)}\right) ;
\end{array}\right] \\
& =2 F_{l: v_{1}+1 ; v_{2} ; \ldots ; v_{n}}^{p: \mu_{1}+1 ; \mu_{2} ; \ldots \mu_{n}}\left[\begin{array}{c}
\left(a_{p}\right): 1+2 r, b_{i}^{(1)}+r,\left(b_{\mu_{1}}^{(1)}\right) ; \ldots ;\left(b_{\mu_{n}}^{(n)}\right) ; \frac{1}{\tau_{1}}, \tau_{2}, \ldots, \tau_{n} \\
\left(\alpha_{l}\right): 1+r,\left(\omega_{v_{1}}^{(1)}\right) ; \ldots ;\left(\omega_{v_{n}}^{(n)}\right) ;
\end{array}\right] .
\end{aligned}
$$

Proof. In view of the derivative operator $r$-times, one finds 


$$
\begin{aligned}
& D_{\tau_{1}}^{r}\left\{\tau_{1}^{-r-1} F_{l: v_{1} ; \ldots ; v_{n}}^{p: \ldots ; \mu_{n}}\left[\begin{array}{l}
\left(a_{p}\right):\left(b_{\mu_{1}}^{(1)}\right) ; \ldots ;\left(b_{\mu_{n}}^{(n)}\right) ; \\
\left.\frac{1}{\tau_{1}}, \ldots, \frac{1}{\tau_{1}}\right] \\
\left(\alpha_{l}\right):\left(\omega_{v_{1}}^{(1)}\right) ; \ldots ;\left(\omega_{v_{n}}^{(n)}\right) ;
\end{array}\right\}\right. \\
& =\sum_{\varsigma_{1}, \ldots, \varsigma_{n}=0}^{\infty} \wedge\left(\varsigma_{1}, \ldots, \varsigma_{n}\right) \frac{\tau_{1}^{-\varsigma_{1}-\varsigma_{2} \cdots-\varsigma_{n}-2 r-1}}{\prod_{i=1}^{n} \varsigma_{i} !}(-1)^{r}\left(\varsigma_{1}+\cdots+\varsigma_{n}+r+1\right)_{r} \\
& =(-1)^{r}(1+r)_{r} \tau_{1}^{-2 r-1} F_{l+1: v_{1} ; \ldots ; v_{n}}^{p+1: \mu_{1} ; \ldots ; \mu_{n}}\left[\begin{array}{c}
1+2 r,\left(a_{p}\right): b_{i}^{(1)}+r,\left(b_{\mu_{1}}^{(1)}\right) ; \ldots ;\left(b_{\mu_{n}}^{(n)}\right) ; \\
1+r,\left(\alpha_{l}\right):\left(\omega_{v_{1}}^{(1)}\right) ; \ldots ;\left(\omega_{v_{n}}^{(n)}\right) ;
\end{array}\right.
\end{aligned}
$$

The application of the Leibnitz formula implies that

$$
\begin{aligned}
& D_{\tau_{1}}^{r}\left\{\begin{array}{l}
\tau_{1}^{-r-1} F_{l: v_{1} ; \ldots ; v_{n}}^{p: \mu_{1} ; ; \mu_{n}}\left[\begin{array}{l}
\left(a_{p}\right):\left(b_{\mu_{1}}^{(1)}\right) ; \ldots ;\left(b_{\mu_{n}}^{(n)}\right) ; \\
\left(\alpha_{l}\right):\left(\omega_{v_{1}}^{(1)}\right) ; \ldots ;\left(\omega_{v_{n}}^{(n)}\right) ;
\end{array} \frac{1}{\tau_{1}}, \ldots, \frac{1}{\tau_{1}}\right]
\end{array}\right\} \\
& =\sum_{k=0}^{r}\left(\begin{array}{c}
r \\
k
\end{array}\right) D_{\tau_{1}}^{r-k}\left\{\tau_{1}^{-r}\right\} D_{\tau_{1}}^{k}\left\{\tau_{1}^{-1} F_{l: v_{1} ; \ldots ; v_{n}}^{p: \ldots ; \mu_{n}}\left[\begin{array}{l}
\left(a_{p}\right):\left(b_{\mu_{1}}^{(1)}\right) ; \ldots ;\left(b_{\mu_{n}}^{(n)}\right) ; \\
\left(\alpha_{l}\right):\left(\omega_{v_{1}}^{(1)}\right) ; \ldots ;\left(\omega_{v_{n}}^{(n)}\right) ;
\end{array} \frac{1}{\tau_{1}}, \ldots, \frac{1}{\tau_{1}}\right]\right\} \\
& =(-1)^{r}(r)_{r} \tau_{1}^{-2 r-1} \sum_{k=0}^{r} \frac{(-r)_{k}}{(1-2 r)_{k}} F_{l+1: v_{1} ; \ldots ; v_{n}}^{p+1: \mu_{1} ; ; \mu_{n}}\left[\begin{array}{c}
1+k,\left(a_{p}\right): b_{i}^{(1)}+r,\left(b_{\mu_{1}}^{(1)}\right) ; \ldots ;\left(b_{\mu_{n}}^{(n)}\right) ; \\
1,\left(\alpha_{l}\right):\left(\omega_{v_{1}}^{(1)}\right) ; \ldots ;\left(\omega_{v_{n}}^{(n)}\right) ;
\end{array} \quad \frac{1}{\tau_{1}}, \ldots, \frac{1}{\tau_{1}}\right] .
\end{aligned}
$$

We combine the above to get (24). Identity (25) is established similarly.

$$
\begin{aligned}
& \sum_{k=0}^{r}\left(\begin{array}{l}
r \\
k
\end{array}\right) \frac{(-1)^{k}(-r)_{k}}{\left(1+a_{i}-r\right)_{k}} F_{l+\alpha: v_{1} ; \ldots ; v_{n}}^{p+\alpha: \mu_{n} ; \ldots, \mu_{n}}\left[\begin{array}{c}
\frac{1+r}{\alpha}, \ldots, \frac{\alpha+r}{\alpha},\left(a_{p}\right):\left(b_{\mu_{1}}^{(1)}\right) ; \ldots ;\left(b_{\mu_{n}}^{(n)}\right) ; \\
\frac{1+r-k}{\alpha}, \ldots, \frac{\alpha+r-k}{\alpha},\left(\alpha_{l}\right):\left(\omega_{v_{1}}^{(1)}\right) ; \ldots ;\left(\omega_{v_{n}}^{(n)}\right) ;
\end{array}\right] \\
& =\frac{(-1)^{r}\left(1+a_{i}\right)_{r}}{\left(-a_{i}\right)_{r}} F_{l+\alpha: \mu_{1} ; \ldots ; v_{n}}^{p+\alpha: \mu_{n}}\left[\begin{array}{c}
\frac{1+a_{i}+r}{\alpha}, \ldots, \frac{\alpha+a_{i}+r}{\alpha},\left(a_{p}\right):\left(b_{\mu_{1}}^{(1)}\right) ; \ldots ;\left(b_{\mu_{n}}^{(n)}\right) ; \\
\frac{1+a_{i}}{\alpha}, \ldots, \frac{\alpha+a_{i}}{\alpha},\left(\alpha_{l}\right):\left(\omega_{v_{1}}^{(1)}\right) ; \ldots ;\left(\omega_{v_{n}}^{(n)}\right) ;
\end{array}\right], \quad \alpha \geq 2,
\end{aligned}
$$

with $i=1, \ldots, p$; 


$$
\begin{aligned}
& \sum_{k=0}^{r}\left(\begin{array}{l}
r \\
k
\end{array}\right) \frac{(-1)^{k}(-r)_{k}}{\left(1+b_{i}^{(1)}-r\right)_{k}} F_{l: v_{1}+\alpha ; v_{2} ; \ldots ; v_{n}}^{p: \mu_{1}+\alpha ; \mu_{2} ; \ldots ; \mu_{n}}\left[\begin{array}{c}
\left(a_{p}\right): \frac{1+r}{\alpha}, \ldots, \frac{\alpha+r}{\alpha}:\left(b_{\mu_{1}}^{(1)}\right) ; \ldots ;\left(b_{\mu_{n}}^{(n)}\right) ; \\
\left(\alpha_{l}\right): \frac{1+r-k}{\alpha}, \ldots, \frac{\alpha+r-k}{\alpha}:\left(\omega_{v_{1}}^{(1)}\right) ; \ldots ;\left(\omega_{v_{n}}^{(n)}\right) ;
\end{array}\right] \\
& \left.=\frac{(-1)^{r}\left(1+b_{i}^{(1)}\right)_{r}}{\left(-b_{i}^{(1)}\right)_{r}} F_{l: v_{1}+\alpha ; v_{2} ; \ldots ; v_{n}}^{p: \mu_{1}+\alpha ; \mu_{2} ; \ldots ; \mu_{n}}\left[\begin{array}{c}
\left(a_{p}\right): \frac{1+b_{i}^{(1)}+r}{\alpha}, \ldots, \frac{\alpha+b_{i}^{(1)}+r}{\alpha},\left(b_{\mu_{1}}^{(1)}\right) ; \ldots ;\left(b_{\mu_{n}}^{(n)}\right) ; \\
\left(\alpha_{l}\right): \frac{1+b_{i}^{(1)}}{\alpha}, \ldots, \frac{\alpha+b_{i}^{(1)}}{\alpha},\left(\omega_{v_{1}}^{(1)}\right) ; \ldots ;\left(\omega_{v_{n}}^{(n)}\right) ;
\end{array}\right] \tau_{1}^{\alpha}, \tau_{2}, \ldots, \tau_{n}\right], \quad \alpha \geq 2,
\end{aligned}
$$

with $i=1, \ldots, \mu_{1}$.

Proof. Firstly, we establish (28). The derivative operator $r$-times leads to

$$
\begin{aligned}
& D_{\tau_{1}}^{r}\left\{\tau_{1}^{a_{i}+r} F_{l: v_{1} ; \ldots ; v_{n}}^{p: \mu_{1} ; \ldots, \mu_{n}}\left[\begin{array}{l}
\left(a_{p}\right):\left(b_{\mu_{1}}^{(1)}\right) ; \ldots ;\left(b_{\mu_{n}}^{(n)}\right) ; \\
\tau_{1}^{\alpha}, \ldots, \tau_{1}^{\alpha} \\
\left(\alpha_{l}\right):\left(\omega_{v_{1}}^{(1)}\right) ; \ldots ;\left(\omega_{v_{n}}^{(n)}\right) ;
\end{array}\right]\right\} \\
& =\sum_{\varsigma_{1}, \ldots, \varsigma_{n}=0}^{\infty} \wedge\left(\varsigma_{1}, \ldots, \varsigma_{n}\right) \frac{\tau_{1}^{\alpha\left(\varsigma_{1}+\cdots+\varsigma_{n}\right)+a_{i}}}{\prod_{i=1}^{n} \varsigma_{i} !} \frac{\left(\alpha\left(\varsigma_{1}+\cdots+\varsigma_{n}\right)+a_{i}+r\right) !}{\left(\alpha\left(\varsigma_{1}+\cdots+\varsigma_{n}\right)+a_{i}\right) !} \\
& =\tau_{1}^{a_{i}} \sum_{\varsigma_{1}, \ldots, \varsigma_{n}=0}^{\infty} \wedge\left(\varsigma_{1}, \ldots, \varsigma_{n}\right) \frac{\tau_{1}^{\alpha\left(\varsigma_{1}+\cdots+\varsigma_{n}\right)}}{\prod_{i=1}^{n} \varsigma_{i}} \frac{\left(a_{i}+r\right) !\left(\left(1+a_{i}+r / \alpha\right), \ldots,\left(\alpha+a_{i}+r / \alpha\right)\right)_{\varsigma_{1}+\cdots+\varsigma_{n}}}{\left(a_{i}\right) !\left(\left(1+a_{i} / \alpha\right), \ldots,\left(\alpha+a_{i} / \alpha\right)\right)_{\varsigma_{1}+\cdots+\varsigma_{n}}} \\
& =\tau_{1}^{a_{i}}\left(1+a_{i}\right)_{r} F_{l+\alpha: v_{1} ; \ldots ; v_{n}}^{p+\alpha, \ldots ; \mu_{n}}\left[\begin{array}{c}
\frac{1+a_{i}+r}{\alpha}, \ldots, \frac{\alpha+a_{i}+r}{\alpha},\left(a_{p}\right):\left(b_{\mu_{1}}^{(1)}\right) ; \ldots ;\left(b_{\mu_{n}}^{(n)}\right) ; \\
\frac{1+a_{i}}{\alpha}, \ldots, \frac{\alpha+a_{i}}{\alpha},\left(\alpha_{l}\right):\left(\omega_{v_{1}}^{(1)}\right) ; \ldots ;\left(\omega_{v_{n}}^{(n)}\right) ;
\end{array}\right] .
\end{aligned}
$$

Again, Leibnitz formula implies that

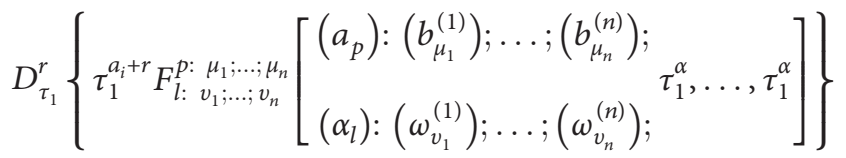

$$
\begin{aligned}
& =\sum_{k=0}^{r}\left(\begin{array}{c}
r \\
k
\end{array}\right) D_{\tau_{1}}^{r-k}\left\{\tau_{1}^{a_{i}}\right\} D_{\tau_{1}}^{k}\left\{\tau_{1}^{r} F_{l: v_{1} ; \ldots ; v_{n}}^{p: \ldots ; \mu_{n}}\left[\begin{array}{c}
\left(a_{p}\right):\left(b_{\mu_{1}}^{(1)}\right) ; \ldots ;\left(b_{\mu_{n}}^{(n)}\right) ; \\
\tau_{1}^{\alpha}, \ldots, \tau_{1}^{\alpha} \\
\left(\alpha_{l}\right):\left(\omega_{v_{1}}^{(1)}\right) ; \ldots ;\left(\omega_{v_{n}}^{(n)}\right) ;
\end{array}\right]\right\}
\end{aligned}
$$

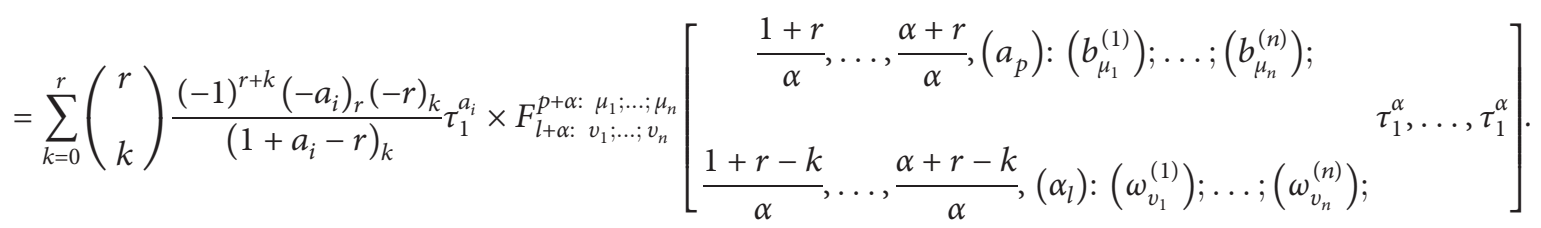

Equating the above two identities yields (28). The other equality (29) can be established similarly. 


\section{FSFs of GKDFS by Rearrangement}

Theorem 9. The following FSFs of GKDFS are verified:

$$
\begin{aligned}
& \sum_{k=0}^{r} \frac{(-1)^{k}\left(a_{i+1}\right)_{k}}{\left(a_{i+1}-a_{i}-r+1\right)_{k}} F_{l: v_{1} ; \ldots ; v_{n}}^{p: \mu_{1} ; \ldots ; \mu_{n}}\left[\begin{array}{c}
a_{i+1}+k,\left(a_{p}^{i+1}\right):\left(b_{\mu_{1}}^{(1)}\right) ; \ldots ;\left(b_{\mu_{n}}^{(n)}\right) ; \tau_{1}, \ldots, \tau_{n} \\
\left(\alpha_{l}\right):\left(\omega_{v_{1}}^{(1)}\right) ; \ldots ;\left(\omega_{v_{n}}^{(n)}\right) ;
\end{array}\right] \\
& \quad=\frac{\left(a_{i}\right)_{r}}{\left(a_{i}-a_{i+1}\right)_{r}} F_{l: v_{1} ; \ldots ; v_{n}}^{p: \mu_{1} ; \ldots ; \mu_{n}}\left[\begin{array}{c}
a_{i}+r,\left(a_{p}^{i}\right):\left(b_{\mu_{1}}^{(1)}\right) ; \ldots ;\left(b_{\mu_{n}}^{(n)}\right) ; \tau_{1}, \ldots, \tau_{n} \\
\left(\alpha_{l}\right):\left(\omega_{v_{1}}^{(1)}\right) ; \ldots ;\left(\omega_{v_{n}}^{(n)}\right) ;
\end{array}\right]
\end{aligned}
$$

with $i=1, \ldots, p-1$;

$$
\begin{gathered}
\sum_{k=0}^{r} \frac{(-1)^{k}\left(b_{i+1}^{(1)}\right)_{k}}{\left(b_{i+1}^{(1)}-b_{i}^{(1)}-r+1\right)_{k}} F_{l: v_{1} ; \ldots ; v_{n}}^{p: \mu_{1} ; \ldots ; \mu_{n}}\left[\begin{array}{c}
\left(a_{p}\right): b_{i+1}^{(1)}+k,\left(b_{\mu_{1}}^{(1), i}\right) ; \ldots ;\left(b_{\mu_{n}}^{(n)}\right) ; \tau_{1}, \ldots, \tau_{n} \\
\left(\alpha_{l}\right):\left(\omega_{v_{1}}^{(1)}\right) ; \ldots ;\left(\omega_{v_{n}}^{(n)}\right) ;
\end{array}\right] \\
=\frac{\left(b_{i}^{(1)}\right)_{r}}{\left(b_{i}^{(1)}-b_{i+1}^{(1)}\right)_{r}} F_{l: v_{1} ; \ldots ; v_{n}}^{p: \mu_{1} ; \ldots ; \mu_{n}}\left[\begin{array}{c}
\left.\left(a_{p}\right): b_{i}^{(1)}+r,\left(b_{\mu_{1}}^{(1), i}\right) ; \ldots ;\left(b_{\mu_{n}}^{(n)}\right) ; \tau_{1}, \ldots, \tau_{n}\right], \\
\left(\alpha_{l}\right):\left(\omega_{v_{1}}^{(1)}\right) ; \ldots ;\left(\omega_{v_{n}}^{(n)}\right) ;
\end{array}\right.
\end{gathered}
$$

with $i=1, \ldots, \mu_{1}-1$.

Proof. In view of the definition of GKDFS, the L. H. S of (32) may be written as

$$
\begin{aligned}
& \sum_{\varsigma_{1}, \ldots, \varsigma_{n}=0}^{\infty} \wedge\left(\varsigma_{1}, \ldots, \varsigma_{n}\right) \prod_{i=1}^{n} \frac{\tau_{i}^{\varsigma_{i}}}{\varsigma_{i} !} F_{1}\left(-r, a_{i+1}+r ; a_{i+1}-a_{i}-r+1 ; 1\right) \\
& =\frac{\left(a_{i}\right)_{r}}{\left(a_{i}-a_{i+1}\right)_{r}} F_{l: v_{1} ; \ldots ; v_{n}}^{p: \mu_{1} \ldots ; \mu_{n}}\left[\begin{array}{c}
a_{i}+r,\left(a_{p}^{i}\right):\left(b_{\mu_{1}}^{(1)}\right) ; \ldots ;\left(b_{\mu_{n}}^{(n)}\right) ; \tau_{1}, \ldots, \tau_{n} \\
\left(\alpha_{l}\right):\left(\omega_{v_{1}}^{(1)}\right) ; \ldots ;\left(\omega_{v_{n}}^{(n)}\right) ;
\end{array}\right] .
\end{aligned}
$$

The application of theorem of Vandermonde,

$$
{ }_{2} F_{1}(-r, a ; b ; 1)=\frac{(b-a)_{r}}{(b)_{r}},
$$

in the above equation gives (32). Result (33) could be obtained in a similar strategy.

\section{Conclusion}

We gave many finite summation identities using the GKDFS. By specializing the parameters in GKDFS, we obtain summation formulas for the generalized Lauricella functions [2, 3], as well as confluent forms of Lauricella series in $n$ variables $\Phi_{2}^{(n)}, \Psi_{2}^{(n)}, \Phi_{D}^{(n)}, \Xi_{1}^{(n)}$, and $\Phi_{3}^{(n)}$ [2]. For instance, characterizing the parameters in (8), we got the finite summation identities for $F_{B}^{(n)}$ and $\Xi_{1}^{(n)}$ :

$$
\begin{aligned}
& \sum_{k=0}^{r}\left(\begin{array}{l}
r \\
k
\end{array}\right) \frac{\left(\xi_{1}\right)_{k}}{(c)_{k}} \tau_{1}^{k} F_{B}^{(n)}\left(\theta_{1}+k, \xi_{1}+k, \xi_{2}, \ldots, \xi_{n} ; c+k ; \tau_{1}, \ldots, \tau_{n}\right)=F_{B}^{(n)}\left(\theta_{1}+r, \xi_{1}, \ldots, \xi_{n} ; c ; \tau_{1}, \ldots, \tau_{1}\right) \\
& \sum_{k=0}^{r}\left(\begin{array}{l}
r \\
k
\end{array}\right) \frac{\left(\xi_{1}\right)_{k}}{(c)_{k}} \tau_{1}^{k} \Xi_{1}^{(n)}\left(\theta_{1}+k, \theta_{2}, \ldots, \theta_{n}, \xi_{1}+k, \xi_{2}, \ldots, \xi_{n-1} ; c+k ; \tau_{1}, \ldots, \tau_{n}\right)=\Xi_{1}^{(n)}\left(\theta_{1}+r, \xi_{1}, \ldots, \xi_{n-1} ; c ; \tau_{1}, \ldots, \tau_{1}\right) .
\end{aligned}
$$


Also, characterizing the parameters in (24), we established the following summation identity for $F_{D}^{(n)}$ and $\Phi_{D}^{(n)}$ :

$$
\begin{aligned}
& \sum_{k=0}^{r} \frac{(-r)_{k}}{(1-2 r)_{k}} F_{D}^{(n)}\left(1+r, \xi_{1}, \ldots, \xi_{n} ; 1 ; \frac{1}{\tau_{1}}, \ldots, \frac{1}{\tau_{1}}\right)=2 F_{D}^{(n)}\left(1+2 r, \xi_{1}, \ldots, \xi_{n} ; 1+r ; \frac{1}{\tau_{1}}, \ldots, \frac{1}{\tau_{1}}\right) \\
& \sum_{k=0}^{r} \frac{(-r)_{k}}{(1-2 r)_{k}} \Phi_{D}^{(n)}\left(1+r, \xi_{1}, \ldots, \xi_{n-1} ; 1 ; \frac{1}{\tau_{1}}, \ldots, \frac{1}{\tau_{1}}\right)=2 \Phi_{D}^{(n)}\left(1+2 r, \xi_{1}, \ldots, \xi_{n-1} ; 1+r ; \frac{1}{\tau_{1}}, \ldots, \frac{1}{\tau_{1}}\right) .
\end{aligned}
$$

\section{Data Availability}

No data were used to support this study.

\section{Conflicts of Interest}

The authors declare that they have no conflicts of interest.

\section{Authors' Contributions}

All authors contributed equally and significantly in writing this article. All authors read and approved the final manuscript.

\section{References}

[1] S. B. Opps, N. Saad, and H. M. Srivastava, "Recursion formulas for Appell's hypergeometric function with some applications to radiation field problems," Applied Mathematics and Computation, vol. 207, no. 2, pp. 545-558, 2009.

[2] H. M. Srivastava and P. W. Karlsson, Multiple Gaussian Hypergeometric Series, John Wiley and Sons, New York, NY, USA, 1985.

[3] H. M. Srivastava and H. L. Manocha, A Treatise on Generating Functions, John Wiley and Sons, New York, NY, USA, 1984.

[4] P. O. Mohammed, H. Aydi, A. Kashuri, Y. S. Hamed, and K. M. Abualnaja, "Midpoint inequalities in fractional calculus defined using positive weighted symmetry function kernels," Symmetry, vol. 13, no. 4, p. 550, 2021.

[5] Y. A. Brychkov and N. Saad, "Some formulas for the Appell functionF1(a, b, b';c; w, z)," Integral Transforms and Special Functions, vol. 23, no. 11, pp. 793-802, 2012.

[6] Y. A. Brychkov and N. Saad, "On some formulas for the Appell functionF2 (a, b, b';c, c';w; z)," Integral Transforms and Special Functions, vol. 25, no. 2, pp. 111-123, 2014.

[7] Y. A. Brychkov and N. Saad, "On some formulas for the Appell functionF3 $\left(\mathrm{a}, \mathrm{a}^{\prime}, \mathrm{b}, \mathrm{b}^{\prime} ; \mathrm{c} ; \mathrm{w}, \mathrm{z}\right)$," Integral Transforms and Special Functions, vol. 26, no. 11, pp. 910-923, 2015.

[8] Y. A. Brychkov and N. Saad, "On some formulas for the Appell function $\mathrm{F} 4\left(\mathrm{a}, \mathrm{b} ; \mathrm{c}, \mathrm{c}^{\prime} ; \mathrm{w}, \mathrm{z}\right)$," Integral Transforms and Special Functions, vol. 28, no. 9, pp. 629-644, 2017.

[9] X. Wang, "Infinite summation formulas of double hypergeometric functions," Integral Transforms and Special Functions, vol. 27, no. 5, pp. 347-364, 2016.

[10] X. Wang and Y. Chen, "Finite summation formulas of double hypergeometric functions," Integral Transforms and Special Functions, vol. 28, pp. 239-253, 2016.

[11] V. Sahai and A. Verma, "Finite summation formulas of Srivastava's general triple hypergeometric function," Asian-
European Journal of Mathematics, vol. 12, p. 15, Article ID 1950020, 2019.

[12] H. M. Srivastava, "Generalized Neumann expansions involving hypergeometric functions," Mathematical Proceedings of the Cambridge Philosophical Society, vol. 63, no. 2, pp. 425-429, 1967.

[13] G. Lauricella, "Sulle funzioni ipergeometriche a piu variabili," Rendiconti del Circolo Matematico di Palermo, vol. 7, no. S1, pp. 111-158, 1893.

[14] H. M. Srivastava, "Hypergeometric functions of three variables," Ganita, vol. 15, pp. 97-108, 1964.

[15] H. M. Srivastava, "Some integrals representing triple hypergeometric functions," Rendiconti del Circolo Matematico di Palermo, vol. 16, no. 1, pp. 99-115, 1967.

[16] E. D. Rainville, Special Functions, Chelsea Publishing Company, New York, NY, USA, 1960. 